Ardit Dvorani*, Constantin Wiesener, Markus Valtin, Hanno Voigt, Andrea Kühn, Nikolaus Wenger and Thomas Schauer

\title{
Mobil4Park: development of a sensor-stimulator network for the therapy of freezing of gait in Parkinson patients
}

https://doi.org/10.1515/cdbme-2020-2013

\begin{abstract}
Parkinson's disease (PD) is one of the most common neurodegenerative diseases in the world which mainly affects the human's motor systems. An estimated number of 7-10 million people worldwide suffer from PD. In Germany, the number of people affected by PD lies at about 300,000 and the number rises every year by approximately 13,000. One of the cardinal symptoms of PD is the freezing of gait (FoG), which arises/appears in the late stages of the PD. FoG is defined as an episodic process with increased restriction of movement or complete blockage despite the intention of moving and, as it can lead to falls and injuries and reduces the quality of life, is considered as one of the most disabling symptoms of PD. In this contribution, we introduce a wearable wireless system designed for gait monitoring and non-invasive electrical stimulation (cueing) in case of a FoG episode.
\end{abstract}

Keywords: electrotactile cueing; freezing of gait; Parkinson's disease; rehabilitation; wearables.

\section{Introduction}

Parkinson's disease (PD) is a chronic neurodegenerative condition, which mainly affects the human motor system. James Parkinson was the first one to describe the disease in 1817 under the name shaking palsy or paralysis agitans [12]. Nowadays the Parkinson's disease is the second most common neurodegenerative disorder with Alzheimer

\footnotetext{
*Corresponding author: Ardit Dvorani, SensorStim Neurotechnology GmbH, Berlin, Germany, E-mail: advorani@sensorstim.com Constantin Wiesener and Hanno Voigt, SensorStim Neurotechnology GmbH, Berlin, Germany

Markus Valtin, Technische Universität Berlin, Control Systems Group, Berlin, Germany

Andrea Kühn and Nikolaus Wenger, Department of Neurology, Charité Universitätsmedizin Berlin, Berlin, Germany

Thomas Schauer, SensorStim Neurotechnology GmbH, Berlin, Germany; Technische Universität Berlin, Control Systems Group, Berlin, Germany
}

taking the first place. The main cause of PD is affiliated with a deficiency of dopamine due to the degeneration of the dopamine-producing nerve cells in the substantia nigra in midbrain. PD is characterized mainly by motor-disabling symptoms like rigidity, bradykinesia, slowness, tremor and freezing of gait (FoG). In addition other non-motordisabling symptoms are observed (e.g. sleep disturbances, depression, psychosis, dementia etc.) [1, 5, 14].

In 2016 the number of patients with PD was estimated to be around 6.1 million worldwide [3]. In addition, the incidence rate of the disease has shown an increasing tendency over the years with 22 per 100,000 person-years for all age groups and up to 529 per 100,000 person-years in the older population over 65 years [8]. The disease represents a major burden for modern society. Due to a gradual and progressive loss of mobility and taking care of oneself, the quality of life, as well as the social life of patients, are affected. Additionally, the treatment costs place a high burden on the health economics $[4,11]$.

One of the cardinal symptoms of PD is FoG. It is a representative sensomotoric symptom of PD observed in the late stages of the disease. It is defined as an episodic process characterized by a complete blockage or severely reduced mobility [16]. A typical freezing episode lasts only a few seconds and rarely extends the $30 \mathrm{~s}$ [17]. The occurrence of FoG episodes increases the risk of falls. Falls represent a major implication as it can lead to physical injuries, fractures, and disabilities. Kalilani et al. [7] estimated that $10.6 \%$ of falls can lead to death.

Continuous monitoring of the gait cycle and FoG episodes, as well as on-demand stimulation, can help the patient in improving the gait. Monitoring the development of gait in PD can provide neurologists with information on the unconstrained environment and physicians on the status of the disease to improve the treatment. Various studies have investigated the effect of rhythmic stimulation in PD patients and reported that visual and auditory stimuli had a positive effect $[2,13,19]$. The studies $[9,15,18]$ reported positive effect using electrical cueing on improving the gait of PD patients.

There exist many studies and research in developing wearables for gait monitoring and detection of FoG events to stimulate the patient to re-initiate the gait. Tay et al. [20] 
presented a system consisting of three inertial sensors positioned at both ankle joints and on the neck. They report the capability of the system in detecting FoG events and stimulating using acoustic and haptic stimuli. Jovanov et al. [6] presented a system consisting of a single inertial sensor and a headphone. The inertial sensor was placed in the foot and was able to detect FoG events using the "freezing index" (FI) introduced by Moore et al. [11]. The detection of FoG episodes triggered acoustic feedback. Mazilu et al. [10] also developed a sensor system consisting of inertial sensors attached at both ankle joints and a smart device. They used a binary classifier for distinguishing between FoG and non-FoG events. The stimulation on detection of FoG events was acoustical.

In this article, we present a wearable wireless system designed for gait monitoring and non-invasive electrical stimulation in case of a FoG episode.

\section{Method}

The proposed sensor-stimulator network is designed to serve as a technical aid for Parkinson patients (see Figures 1 and 3). The system consists of two integrated sensors, that can be positioned for example on the instep at the shoe and the shank in a cuff, a five-channel electrical stimulator, and a smart device. Each sensor consists of an inertial measurement unit (IMU) and a biosignal data acquisition interface for two electromyografie (EMG) channels. The IMUs are used for gait performance monitoring and real-time gait phase detection. The IMUs integrate a 12 bit 3D accelerometer, a 16 bit 3D gyroscope and a 12 bit 3D magnetometer and can record the kinematic measures with a data rate of up to $200 \mathrm{~Hz}$. Both sensors are based on an ARM CORTEX-M4 microcontroller, which has enough computing power to enable the implementation of complex algorithms. Taking advantage of the calculating power of the used microcontroller, on both sensor nodes algorithms for real-time gait phase detection, orientation and step

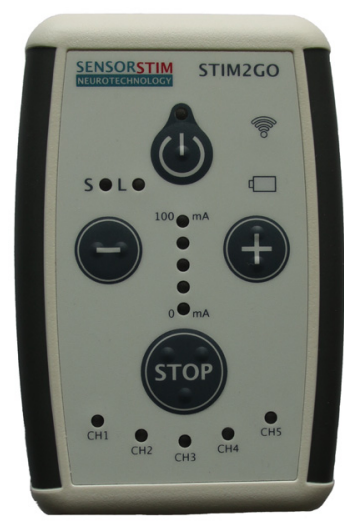

(a) Stimulator

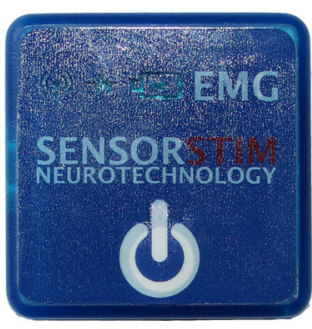

(b) Integrated sensor
Figure 1: Developed stimulator and inertial measurement unit (IMU)/EMG sensor. length estimation are running. The outputs of these algorithms and the EMG data from the leg musculature (dorsi and plantar flexors) can be further processed and used as features for detecting FoG episodes.

The stimulator is positioned on the hip using a belt clip. Same as the sensor node, the computing core is represented by an ARM CORTEX-M4 microcontroller. In addition, a second dedicated microcontroller is responsible for the stimulation. The stimulator can deliver on trigger electrical pulses to designated muscles with the aim to unfreeze the blockade and to reinitialize the gait. One possible stimulation site is the peroneus nerve and the muscle tibialis anterior as commonly used in drop foot stimulation with stroke patients. A special cuff was designed to host the shank IMU/EMG sensor and to attach the stimulation and EMG measurement electrodes below the knee at the shank. The information on the current gait phase delivered from the sensor nodes enables to synchronize the stimulation with the attempt to lift the heel for swing phase initiation. In the stimulator, another IMU is integrated as well as a haptic device for tactile cueing. It allows for a different kind of stimulation. One possible scenario is the use of tactile cueing for preventing the occurrence of a FoG event and the use of electrical stimulation to unfreeze an FoG episode. When the electrical stimulation represents only a sensory stimulation, there exists no risk of falling as a result of stimulation. A smart device is used for data visualization, the configuration of network nodes, i.e. regulating the stimulation intensity, etc., and data logging. For communication in the context of telemedicine, the smart device can be used as an interface for cloud services. The logged data during daily activities could be made available to physicians. In such a way physicians could track the development of the PD and make corresponding changes to the patient's treatment. The communication between the network nodes is realized via Bluetooth Low Energy (BLE). The topology of the wireless sensor network depends on the operating mode of the system (See Figure 2). Two modes are implemented for the network, cueing and logging. Cueing is realized in the star topology with the stimulator as a router. The sensor nodes communicate directly with the

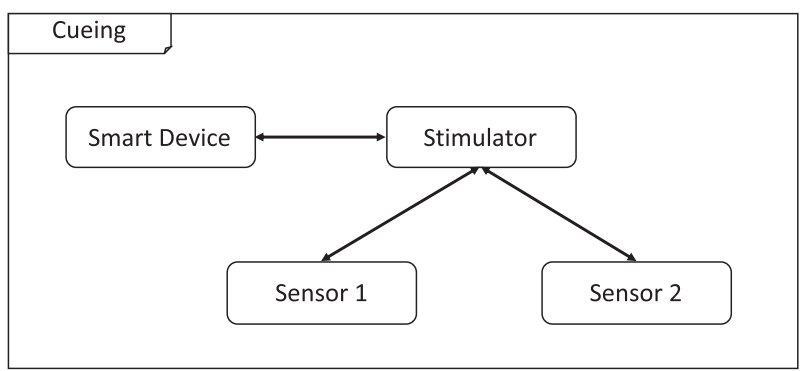

(a) Network topology in Cueing operating mode

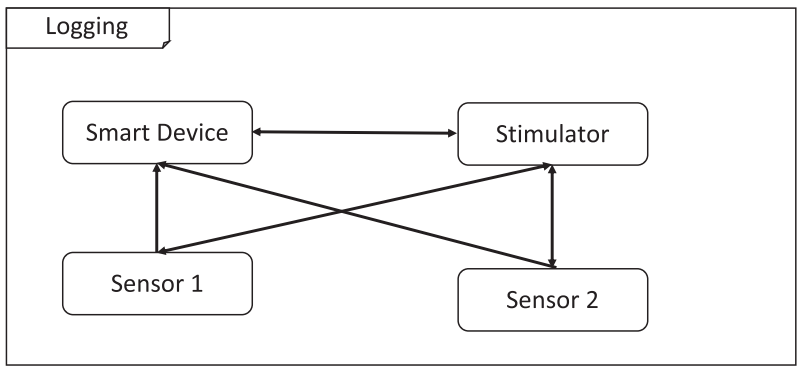

(b) Network topology in Logging operating mode

Figure 2: Network topologies. 


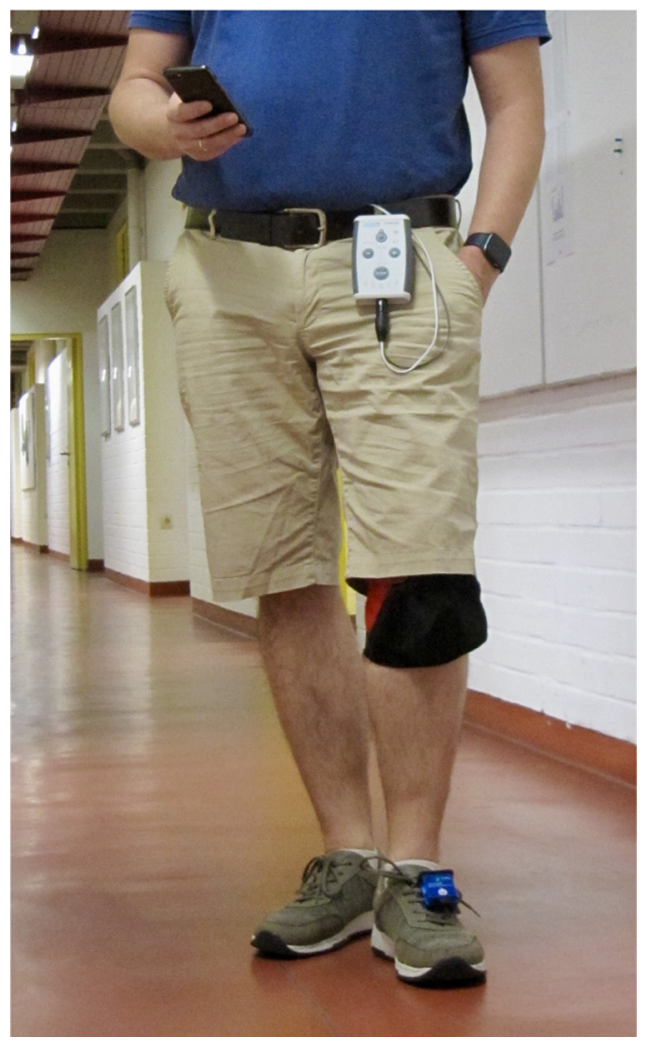

Figure 3: System components on a test subject: IMU/EMG sensors on the left foot and shank (in the cuff), electrical stimulator at the hip and smart devices (phone and watch) as user interfaces. The most affected leg of a Parkinson patient will be used for FoG detection and stimulation.

stimulator and the stimulator acts as an intermediate between the smart devices and sensor nodes. This operating mode is characterized by low data traffic as only gait descriptive features are transmitted for every gait phase and cycle from the sensor nodes. Raw sensor data can be optionally stored on SD-cards within the sensors for later PC-based offline processing. In the case of logging (streaming) mode, a mesh topology is realized. Raw data are transmitted (streamed) in real-time to the smart device and/or to the stimulator. The logging takes place on the smart device. High data traffic characterizes this mode.

\section{Results}

The system has been designed, and the software and hardware have been realized. It can detect in real-time with very high accuracy gait phases exploiting the kinematic measurement of mid-foot and shank. The use of an adaptive network topology depending on the operating mode of the system helps in achieving minimal latency time. The average communication latency between the sensor nodes and the stimulator is $7.5 \mathrm{~ms}$. Adapting the network topology to the two different operating modes yields a minimal latency in the cueing mode as well as a high data throughput in the logging (streaming) mode. The use of the BLE standard saves energy and enables an operating time of over $10 \mathrm{~h}$.

\section{Conclusion}

In this article, we introduced a wearable system for gait monitoring and stimulation. We were able to design an adaptive wireless sensor network topology. The system is able to detect the gait phases in real-time and extract features from the profile of the gait cycle. The data is delivered to a smart device and stimulator with minimal latency. Detecting the gait phase in real-time allows the system to stimulate at the right moment in order to improve the gait. The next step is the development of FoG detection and prediction based on the extracted features and expert labelled patient data. After that, a clinical study will be carried out to investigate the effects of the system on FoG in PD. An extension of the system for up to five sensors is currently under investigation.

Acknowledgement: This work was funded by the German Federal Ministry of Education and Research (BMBF) within the project Mobil4Park (FKZ 16SV8168).

Research funding: This work was funded by the German Federal Ministry of Education and Research (BMBF) within the project Mobil4Park (FKZ 16SV8168).

Author contributions: All authors have accepted responsibility for the entire content of this manuscript and approved its submission.

Competing interests: $\mathrm{AD}, \mathrm{CW}$ and $\mathrm{HV}$ are employees and TS is scientific adviser of the SensorStim Neurotechnology $\mathrm{GmbH}$, which is a company developing sensor-based stimulation devices. All other authors declare that they have no competing interests.

Informed consent: Informed consent was obtained from all individuals included in this study.

Ethical approval: Research involving human subjects complied with all relevant national regulations, institutional policies and is in accordance with the tenets of the Helsinki Declaration, and has been approved by the authors' Institutional Review Board or equivalent committee.

\section{References}

1. Davie CA. A review of Parkinson's disease. Br Med Bull June 2008; 86:109-27.

2. Donovan S, Lim C, Diaz N, Browner N, Rose P, Sudarsky LR, et al. Laserlight cues for gait freezing in Parkinson's disease: an openlabel study. Park Relat Disord May 2011;17:240-5. 
3. Dorsey ER, Elbaz A, Nichols E, Abd-Allah F, Abdelalim A, Adsuar JC, et al. Global, regional, and national burden of Parkinson's disease, 1990-2016: a systematic analysis for the global burden of disease study 2016. Lancet Neurol November 2018;17:939-53.

4. Giladi N, Hausdorff JM. The role of mental function in the pathogenesis of freezing of gait in Parkinson's disease. J Neurol Sci October 2006;248:173-6.

5. Jankovic J. Parkinson's disease: clinical features and diagnosis. J Neurol Neurosurg Psychiatr April 2008;79:368-76.

6. Jovanov E, Wang E, Verhagen L, Fredrickson M, Fratangelo R. deFOG - a real time system for detection and unfreezing of gait of Parkinson's patients. In: Proceedings of Annual International Conference of the IEEE Engineering in Medicine and Biology Society, 2009. IEEE, Minneapolis, MN, USA; September 2009. pp. 5151-4.

7. Kalilani L, Asgharnejad M, Palokangas T, Durgin T. Comparing the incidence of falls/fractures in Parkinson's disease patients in the US population. PloS One 2016;11:e0161689.

8. Lill CM, Klein C. Epidemiologie und Ursachen derÂ ParkinsonErkrankung. Nervenarzt April 2017;88:345-55.

9. Mann GE, Finn SM, Taylor PN. A pilot study to investigate the feasibility of electrical stimulation to assist gait in Parkinson's disease. Neuromodulation: Technol Neural Interface 2008;11:143-9.

10. Mazilu S, Hardegger M, Zhu Z, Roggen D, Tröster G, Plotnik M, et al. Online detection of freezing of gait with smartphones and machine learning techniques. In: Proceedings of the 6 th International Conference on Pervasive Computing Technologies for Healthcare (PervasiveHealth 2012). IEEE, San Diego, California; May 2012.

11. Moore O, Peretz C, Giladi N. Freezing of gait affects quality of life of peoples with Parkinson's disease beyond its relationships with mobility and gait. Mov Disord 2007;22:2192-5.
12. Parkinson J. An essay on the shaking palsy. J Neuropsychiatry Clin Neurosci 2002;14:223-36.

13. Picelli A, Camin M, Tinazzi M, Vangelista A, Cosentino A, Fiaschi A, et al. Three-dimensional motion analysis of the effects of auditory cueing on gait pattern in patients with Parkinson's disease: a preliminary investigation. Neurol Sci August 2010;31:423-30.

14. Poewe W. Non-motor symptoms in Parkinson's disease. Eur J Neurol 2008;15:14-20.

15. Popa L, Taylor P. Functional electrical stimulation may reduce bradykinesia in Parkinson's disease: a feasibility study. J Rehabil Assist Technol Eng October 2015;2. https://doi.org/10.1177/ 2055668315607836.

16. Punin C, Barzallo B, Clotet R, Bermeo A, Bravo M, Bermeo JP, et al. A non-invasive medical device for Parkinson's patients with episodes of freezing of gait. Sensors January 2019;19:737.

17. Schaafsma JD, Balash Y, Gurevich T, Bartels AL, Hausdorff JM, Giladi N. Characterization of freezing of gait subtypes and the response of each to levodopa in Parkinson's disease. Eur J Neurol 2003;10:391-8.

18. Sijobert B, Denys J, Coste CA, Geny C. IMU based detection of freezing of gait and festination in Parkinson's disease. In: Proceedings of the 19th IEEE International Functional Electrical Stimulation Society Annual Conference (IFESS). IEEE, Kuala Lumpur, Malaysia; September 2014. pp. 1-3.

19. Suteerawattananon M, Morris GS, Etnyre BR, Jankovic J, Protas EJ. Effects of visual and auditory cues on gait in individuals with Parkinson's disease. J Neurol Sci April 2004;219:63-9.

20. Tay A, Yen SC, Lee PY, Wang CY, Neo A, Phan SW, et al. Freezing of gait (FoG) detection for Parkinson disease. In: Proceedings of the 10th Asian Control Conference (ASCC). IEEE, Kota Kinabalu, Malaysia; May 2015. pp. 1-6. 